\title{
Morpho-Physiological approach to the paracellular route for salivary secretion by isolated perfused submandi- bular gland
}

\author{
Masataka Murakami ${ }^{1}$, Sadamitsu Hashimoto ${ }^{2}$, Muxin $\mathrm{Wei}^{3}$, and Adrian E Hill ${ }^{4}$ \\ 'Nano-structure Physiology, National Institute for Physiological Sciences, OIB, National Institutes of \\ Natural Sciences, Myoudaiji, Okazaki, Japan ; Department of Pathology, hrc7, Tokyo Dental College, \\ Chiba, Japan ; ${ }^{3}$ Department of Chinese Medicine, Nanjing Medical University, Nanjing, China ; and \\ ${ }^{4}$ Physiological Laboratory, University of Cambridge, Cambridge, UK.
}

\begin{abstract}
A part of the salivary components are shifted from the blood via a trans- and/ or paracellular route. The isolated, arterially perfused, salivary glands (submandibular and parotid glands) were used to assess paracellular transport functionally and morphologically. In the present study, the hydrostatic pressure of the perfusion was changed and the fluid secretion and paracellular transport of fluorescent dye in the isolated perfused submandibular gland were examined. The present findings lead to the conclusion that part of the paracellular transport could be driven by hydrostatic pressure, and that fluid movement drags the solutes. J. Med. Invest. 56 Suppl. : 322-324, December, 2009
\end{abstract}

Keywords : salivary secretion, submandibular salivary gland, fluid secretion, paracellular transport, driving force

\section{A SHORT REVIEW}

A part of the salivary components are shifted from the blood via a paracellular route. The isolated, arterially perfused, salivary glands (submandibular and parotid glands) were used to assess paracellular transport functionally and morphologically after stimulation with carbamylcholine (CCh). Various sizes of ${ }^{3} \mathrm{H}$-labeled dextrans were added to the perfusate and the size of secreted dextrans and their concentration compared. The results revealed two paracellular routes : a narrow route to allow passage of small molecules less than $5 \AA$ radius, and a wider route (1).

Received for publication October 21, 2009 ; accepted October 28, 2009.

Address correspondence and reprint requests to Masataka Murakami, Nano-structure Physiology, National Institute for Physiological Sciences, Myoudaiji, Okazaki 444-8787, Japan and Fax : +81-564-59-5269.
Without any stimulation, Lucifer Yellow (LY), a fluorescent dye, could not enter the luminal space of whole glands beyond the tight junction (intercellular canalliculi, IC) from the perfusate. However, isolated acini under observation by a confocal laser microscope, treated with collagenase allowed the LY entry into the IC. CCh stimulates both trans- and paracellular fluid secretion. Fluorescence intensity in the IC fell during CCh stimulation. If this is assumed to be due to dilution only by transcellular fluid secretion, the paracellular component of fluid secretion was estimated to be at least $60 \%$ of the total fluid secretion (2).

Isoproterenol (Isp) increased the LY secretion. A freeze-fracture replica was prepared by rapid freezing by liquid He. During CCh-Isp stimulation the meshwork of cytoskeleton underlying the tight junction was shrunken (3). Hyperosmotic challenge with sucrose in isolated rat submandibular gland decreased fluid secretion. The decrement 
in normal rats was bigger than in rats extremely-low in aquaporin-5 (AQP5), suggesting AQP5-mediated control of junctional flow (4).

A Chinese herb, Danshen (DS), induced a slow rise of fluid secretion (5). The LY secretion also increased in parallel to fluid secretion during application of DS, suggesting DS could open the junctions.

\section{INTRODUCTION AND PURPOSE OF THE PRESENT STUDY}

Imai (1973) reported a linear relationship between hydrostatic pressure and fluid secretion rate of the in situ submandibular gland under electrical stimulation of chorda tympani. From the slope of the line, he estimated the single hydraulic conductance across exocrine epithelium and that a sum of the osmotic pressure and the hydrostatic pressure differences across epithelium should be a driving force of fluid secretion (6). In the present study, we examined whether the hydrostatic pressure influences the fluid secretion rate and the LY secretion by the isolated perfused submandibular gland of the rat.

\section{METHODS}

Under anesthesia with penthobarbitone sodium (50 $\mathrm{mg} / \mathrm{kg}$ body weight) the submandibular gland was surgically isolated from the male rat of Wistar strain (270-290 $\mathrm{g}$ body weight). The feeding artery was cannulated and vascularly perfused with a peristaltic pump (Cole-Palmer). The excretory duct was also cannulated with a fluorinated fiber tube (OD x ID $=0.5 \mathrm{~mm} \times 0.3 \mathrm{~mm}$, EXLON, IWAKI). A pressure transducer (AD Instruments) was attached to the metal block at the arterial side used for keeping the temperature the same as the perfused gland. To assess the paracellular transport, LY (Molecular Probes) was added and the concentration in the saliva was measured by use of a microplate reader (Beckman). Secretion was induced by 0.1-1.0 $\mu \mathrm{M}$ CCh (Sigma). The arterial pressure was chosen by changing the pump rate.

\section{RESULTS AND DISCUSSION}

At the pump rate of $1.8 \mathrm{ml} / \mathrm{min}$, the arterial pressure was $147 \pm 5 \mathrm{mmHg}(\mathrm{SEM}, \mathrm{n}=26)$. By addition of $1.0 \mu \mathrm{M} \mathrm{CCh}$ to induce salivary secretion the arterial pressure was reduced to $103 \pm 5 \mathrm{mmHg}$ (SEM, $\mathrm{n}=18$ ). Washout of CCh caused the pressure to return to control in around $25 \mathrm{~min}$. Addition of LNAME $(0.3 \mathrm{mM})$, an inhibitor of NO synthase increased the pressure to $180 \pm 13 \mathrm{mmHg}$ (SEM, $\mathrm{n}=$ 10) in $10 \mathrm{~min}$. Addition of $\mathrm{CCh}$ on perfusion with LNAME reduced the pressure to $111 \pm 9 \mathrm{mmHg}$ $(\mathrm{SEM}, \mathrm{n}=10)$. The observation indicated that the pressure during secretion was not affected by NO reduction. The arterial pressure was measured at different pump rates under different concentration of $\mathrm{CCh}$. Because the pump rate and arterial pressure are in a linear relationship, we obtained the arterial resistance from the slope. The arterial resistance is determined by the arteriolar resistance and that of the arterio-venous shunt. At $\mathrm{CCh}=0$, the pressure was $59 \pm 5 \mathrm{mmHg}(\mathrm{SEM}, \mathrm{n}=6)$; at $\mathrm{CCh}=0.5,46 \pm$ $2 \mathrm{mmHg}(\mathrm{SEM}, \mathrm{n}=6)$. The pressure at 0 flow rate was extrapolated to around $25 \mathrm{mmHg}$.

During stimulation with $\mathrm{CCh}$, the LY secretion varied in proportion to the pump rate. On the other hand, the fluid secretion increased or decreased according to the rise or fall of the perfusion rate, but the changed value was not proportional to the pressure changes. The present findings indicate that a part of the paracellular transport could be driven by hydrostatic pressure, and fluid movement drags the solutes.

\section{REFERENCES}

1) Murakami M, Shachar-Hill B, Steward MC, Hill AE : The paracellular component of water flow in the rat submandibular gland. J Physiol $537: 899-906,2001$

2) Segawa A, Yamashina S, Murakami M : Visualization of 'water secretion' by confocal microscopy in rat salivary glands : possible distinction of para- and transcellular pathway. Eur J Morphol 40 : 241-246, 2002

3) Hashimoto S, Murakami M, Kanaseki T, Kobayashi S, Matsuki M, Shimono M, Segawa A. Morphological and functional changes in cell junctions during secretory stimulation in the perfused submandibular gland. Eur J Morphol $41: 35-39,2003$

4) Murakami M, Murdiastuti K, Hosoi K, Hill AE : $\mathrm{AQP}$ and the control of fluid transport in a salivary gland. J Memb Biol 210 : 91-103, 2006

5) Murakami M, Wei M, Ding W, Zhang Q : Effects of chinese herbs on salivary fluid secretion 
by isolated and perfused rat submandibular glands. World J Gastroentenol 15 : 3908-3915, 2009

6) Imai $Y$ : Evidence for the osmotic flow across dog submaxillary gland epithelia as a cause of salivary secretion. Jpn J Physiol 23 : 635-644, 1973 